\title{
Erythema annulare centrifugum?
}

\section{Erythema annulare centrifugum?}

Autoren

Institute
M. Ziemer ${ }^{1}$, K. Eisendle ${ }^{2}$, B. Zelger ${ }^{2}$

Klinik für Dermatologie und Dermatologische Allergologie, Friedrich Schiller Universität, Jena

2 Klinik für Dermatologie und Venerologie, Medizinische Universität Innsbruck, Österreich

\section{Bibliografie}

DOI http://dx.doi.org/

10.1055/s-0029-1243851

Online-Publikation: 25. 1.2010

Akt Dermatol 2010; 36:

222-225 @ Georg Thieme

Verlag KG Stuttgart · New York ISSN 0340-2541

\section{Korrespondenzadresse} Dr. med. Mirjana Ziemer Klinik für Dermatologie und Dermatologische Allergologie Friedrich Schiller Universität Erfurter Str. 35

07743 Jena

mirjana.ziemer@

derma.uni-jena.de

\section{Zusammenfassung \\ $\nabla$}

Das Erythema annulare centrifugum (EAC) ist ein klinisches Reaktionsmuster und verkörpert keine spezifische klinisch-histologische Entität [1,2]. Wie der vorliegende Fall eines EAC-artigen streuenden allergischen Kontaktekzems veranschaulicht, können sich hinter dem Begriff EAC verschiedene distinkte Diagnosen verbergen. Diese Beobachtung konnte inzwischen durch eine umfangreiche retrospektive Analyse von so genannten EAC-Fällen bestätigt werden. Schlussfolgernd,

\section{Einführung}

Das Erythema annulare centrifugum (EAC) wird in den meisten Lehrbüchern als eine entzündliche Dermatose ungeklärter Ätiologie und als Hauptvertreter der „Figurierten Erytheme“ dargestellt [3]. Zu Beginn, so die Ausführungen verschiedenster Autoren, präsentieren sich erythematöse Makulae oder urtikarielle Papeln, die sich langsam zentrifugal ausbreiten und zentral aufhellen, sodass ein annulärer Eindruck entsteht [4]. Konfluierende Läsionen bilden bogenförmige oder polyzyklische Herde [4]. Eine derartige Definition triff jedoch tatsächlich für eine Vielzahl von Dermatosen mit annulärer Morphe zu. So umfasst die Gruppe der „Figurierten Erytheme“ in den Lehrbüchern neben dem EAC auch solche heterogenen Erkrankungen wie das Erythema marginatum und Erythema gyratum repens. Zugleich können sich mit figurierten Läsionen vielzählige weitere spezifische Dermatosen präsentieren wie eine annuläre Urtikaria, Tinea corporis, annuläre Varianten einer Psoriasis oder Mycosis fungoides, aber auch ein Lupus erythematosus (LE). Bereits vor einigen Jahren erschienen Publikationen zum EAC, die darlegten, dass sich hinter diesem Begriff verschiedene Erkrankungen verbergen. Aktuelle Untersuchungen konnten nachweisen, dass es muss die klinische und histologische Differenzialdiagnose „EAC“ dazu führen, wenigstens drei Hauptgruppen an Erkrankungen in Erwägung zu ziehen, die sich häufig dahinter verbergen. Neben einem (tumiden) Lupus erythematosus handelt es sich dabei um Fälle spongiotischer Dermatitiden entweder im Sinne einer subakuten und chronischen Dermatitis („Ekzem“), exzessiver Varianten einer Pityriasis rosea oder Stasis-Dermatitis. Darüber hinaus, weniger häufig, liegen Pseudolymphome, insbesondere Borrelien-assoziierte Pseudolymphome vor.

sich beim EAC um einen Sammelbegriff für verschiedenste klinisch-histologische Reaktionsmuster handelt, hinter denen sich neben Varianten eines LE vor allem spongiotische Dermatitiden vom Ekzemtyp und der Piytriasis rosea (PR) aber auch Pseudolymphome, darunter Borrelienassoziierte verbergen [5]. Die folgende Kasuistik soll das einmal mehr verdeutlichen.

\section{Kasuistik}

$\nabla$

Ein 61-jähriger Patient stellte sich erstmalig Anfang August 2006 in unserer Klinik vor. Er beobachtete am rechten Unterschenkel eine stark juckende Rötung seit ca. Mitte Juli 2006, welche er mit einem Stoß in Zusammenhang bringt. In diesem Zusammenhang kam auch eine „Salbe“ zur Anwendung, an deren Namen sich der Patient jedoch nicht erinnert. Im Verlauf der nächsten Wochen kam es zur Ausbreitung der Hautveränderungen auf den linken Unterschenkel, die Innenseiten beider Oberschenkel, das Gesäß sowie den kaudalen Teil des Rumpfs.

Nebendiagnosen sind ein Diabetes mellitus Typ 2, eine koronare Herzkrankheit (Z.n. Bypass) und Herzinsuffizienz sowie eine periphere arterielle Verschlusskrankheit. Ödeme beider Unterschen- 
kel bestehen kardial bedingt seit mehreren Jahren. Der Patient gibt zudem ein Epilepsieleiden an, ist aber seit ca. 4 Jahren anfallsfrei. In diesen Zusammenhängen besteht eine umfangreiche Medikation, die jedoch seit längerem unverändert war.

Bei der klinischen Untersuchung zeigt sich ein flächiges, unscharf begrenztes Erythem an beiden Unterschenkeln mit Übergang auf die Oberschenkelinnenseiten ( $\bullet$ Abb. 1). Darüber hinaus finden sich vielzählige erythematöse, teils konfluente, leicht infiltrierte Papeln und Plaques. Die größeren annulären Läsionen zeigen eine deutliche Randbetonung und eine periphere, nach innen gerichtete Schuppenkrause ( $\bullet$ Abb. 1).

Die Histologie einer Hautbiopsie vom Oberschenkel weist weitgehend eine korbgeflechtartige Orthokeratose jedoch mit fokalen Parakeratosehügeln auf ( $\bullet$ Abb.2 und 3). Multifokal zeigt sich eine Spongiose bis hin zur Ausbildung kleiner spongiotischer Bläschen. In diesen Abschnitten kommt es zur Exozytose von Lymphozyten in die Epidermis. In der angrenzenden Dermis findet sich ein oberflächliches, vorwiegend perivaskuläres, lymphozytäres Infiltrat mit einzelnen eosinophilen Granulozyten und einzelnen Erythrozytenextravasaten ( $\boldsymbol{Q}$ Abb. 3). Zusammenfassend zeigt sich eine akute spongiotische Dermatitis.

Die Behandlung erfolgte bei Verdacht auf ein allergisches Kontaktekzem des Unterschenkels mit Streuung. Eine Atopiediagnostik war unauffällig. Über 2 Tage verabreichten wir $40 \mathrm{mg}$ Prednisolon. Extern kam zunächst Fucicort-Creme, später dann Laticort 0,1\%-Salbe zur Anwendung. Unter dieser Therapie zeigte sich eine allmähliche und anhaltende Befundverbesserung. $\mathrm{Zu}$ den angeratenen allergologischen Testungen hat sich der Patient im Verlauf leider nicht mehr vorgestellt.

\section{Diskussion \\ $\nabla$}

Das EAC wurde erstmals 1916 von Jean Darier publiziert. Er beschrieb das EAC als selbstlimitierte Dermatose mit indurierten, annulären Läsionen ohne epidermale Beteiligung, die sich zügig ausbreiten und nach 1-2 Wochen spontan zurückbilden, während gleichzeitig neue Läsionen entstehen. Histologisch beschrieb Darier eine normale Epidermis und ein oberflächliches und tiefes Infiltrat aus Lymphozyten und Makrophagen [6].

Heute wird das EAC in Standardlehrbüchern neben dem Erythema marginatum und Erythema gyratum repens der heterogenen Gruppe „Figurierter Erytheme“ zugeordnet, die sich lediglich durch ihre überlappende morphologische Charakteristik langsam migrierender, annulärer bis figurierter Erytheme mit oder ohne periphere Schuppung auszeichnet [7]. Annuläre erythematöse Läsionen treten jedoch darüber hinaus auch beim LE, bei annulärer Urtikaria, einer Tinea corporis und bei annulären Varianten einer Psoriasis oder Mycosis fungoides auf und sind daher durchaus mit einem so definierten EAC zu verwechseln.

Auch histologisch werden Fälle eines EAC - im Kontrast zu Dariers Original-Beschreibung - mit heterogenen Veränderungen präsentiert. Unterschieden wurden zuletzt eine oberflächliche und eine tiefe Variante eines EAC $[2,8]$. Ackerman schlägt für die oberflächliche schuppende Variante den Begriff „superficial figurate erythema" vor und separiert dieses von dem bei Darier beschriebenen „tiefen Typ“ [8]. Ein oberflächliches, perivaskuläres, lymphozytäres Infiltrat mit gelegentlichem Vorhandensein einiger eosinophiler Granulozyten wird begleitet durch eine herdförmige Spongiose und kleine Parakeratosehügel. Die histologischen Veränderungen des oberflächlichen Typs sind jedoch nicht spezifisch und entsprechen im Wesentlichen einer spongiotischen

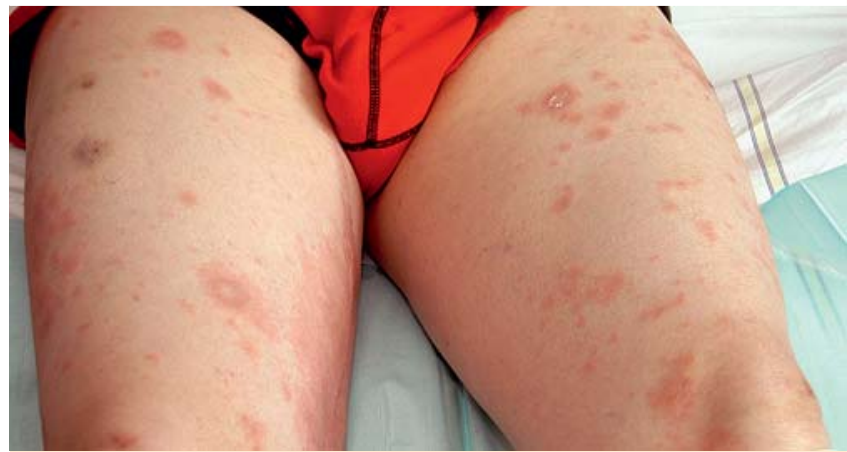

Abb. 1 Flächiges unscharf begrenztes Erythem an den Oberschenkelinnenseiten. Darüber hinaus vielzählige erythematöse, teils konfluente, leicht infiltrierte Papeln und annuläre Plaques. Annuläre Läsionen mit Randbetonung und einer peripheren, nach innen gerichteten Schuppenkrause.

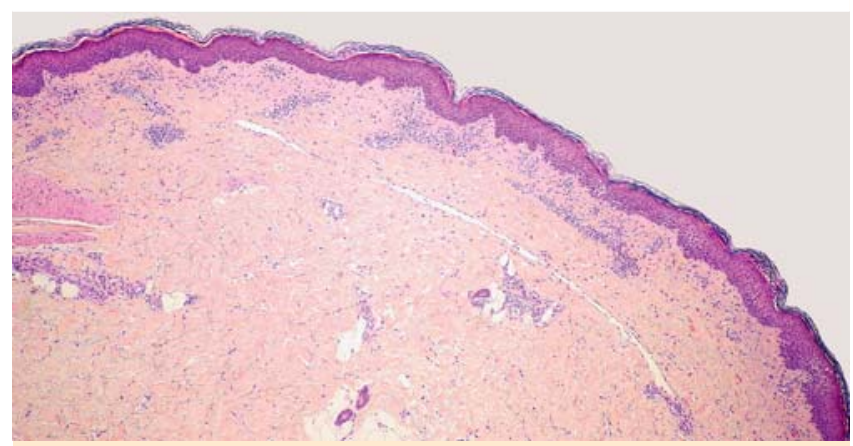

Abb. 2 Multifokale Spongiose. Dermales oberflächliches, vorwiegend perivaskuläres, lymphozytäres Infiltrat (Hämatoxylin-Eosin, Originalvergrößerung $\times 40$ ).

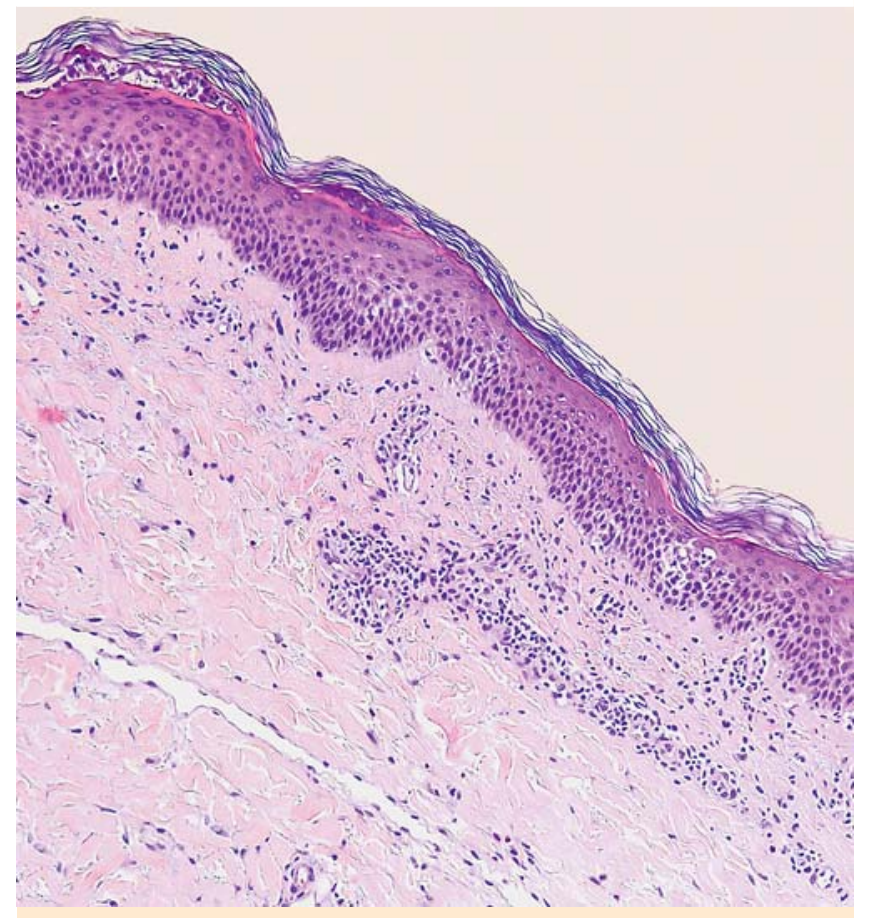

Abb. 3 Korbgeflechtartige Orthokeratose mit fokalen Parakeratosehügeln. Exozytose von Lymphozyten in die spongiotischen Epidermisabschnitte. Im dermalen Infiltrat einzelne eosinophile Granulozyten und einzelne Erythrozytenextravasate (Hämatoxylin-Eosin, Originalvergrößerung $\times 200$ ). 
Tab. 1 Klinisch-histologische Differenzierung des EAC-Reaktionsmusters [5].

\begin{tabular}{|c|c|c|c|}
\hline & Lupus erythematosus & Spongiotische Dermatitis & $\begin{array}{l}\text { (Borrelien-assoziierte) } \\
\text { Pseudolymphome }\end{array}$ \\
\hline Alter & zumeist jüngere Frauen & $\begin{array}{l}\text { häufiger Männer; in der Regel } \\
10-20 \text { Jahre älter als die } \\
\text { LE-Patienten }\end{array}$ & mehr erwachsene Frauen \\
\hline Lokalisation & meistens Rumpf und Gesicht & $\begin{array}{l}\text { meistens Rumpf und untere } \\
\text { Extremitäten }\end{array}$ & $\begin{array}{l}\text { meistens Rumpf und untere } \\
\text { Extremitäten }\end{array}$ \\
\hline $\begin{array}{l}\text { Charakteristische } \\
\text { klinische Aspekte }\end{array}$ & $\begin{array}{l}\text { einzelne Läsionen, zentrale grau-livide Verfärbung, } \\
\text { leichte epidermale Atrophie, jedoch keine Schuppung } \\
\text { oder Krusten }\end{array}$ & $\begin{array}{l}\text { multiple schuppende Läsionen, } \\
\text { die unterschiedlich eine atopi- } \\
\text { sche Dermatitis, Stasis-Derma- } \\
\text { titis oder irritierte Pityriasis } \\
\text { rosea begleiten }\end{array}$ & $\begin{array}{l}\text { bläulich-pseudolymphomatöser } \\
\text { Aspekt, keine epidermale Betei- } \\
\text { ligung, keine zentrale Verfär- } \\
\text { bung, Migrationstendenz }\end{array}$ \\
\hline $\begin{array}{l}\text { Histologische } \\
\text { Veränderungen }\end{array}$ & $\begin{array}{ll}\begin{array}{l}\text { oberflächliche und } \\
\text { tiefe, perivaskuläre } \\
\text { lymphozytäre Infil- } \\
\text { trate und Muzin }\end{array} & \begin{array}{l}\text { dichte lymphozytäre Man- } \\
\text { schetten um dilatierte post- } \\
\text { kapilläre Venolen des super- } \\
\text { fiziellen Gefäßplexus }\end{array} \\
\begin{array}{l}\text { In einigen Fällen diskrete vakuoläre Degeneration } \\
\text { entlang der epidermo-dermalen Junktion oder entlang } \\
\text { der Akrosyringia und Infundibula }\end{array}\end{array}$ & $\begin{array}{l}\text { Spongiose und Parakeratose } \\
\text { eher spärliches bis moderates } \\
\text { oberflächliches perivaskuläres } \\
\text { lymphozytäres Infiltrat, } \\
\text { gelegentlich eosinophile } \\
\text { Granulozyten } \\
\text { diskretes Ödem in der papil- } \\
\text { lären Dermis, mitunter wenige } \\
\text { extravasierte Erythrozyten }\end{array}$ & $\begin{array}{l}\text { keine epidermalen Verände- } \\
\text { rungen } \\
\text { oberflächliche und tiefe lympho- } \\
\text { zytäre Infiltrate mit unterschied- } \\
\text { licher Beimengung von Plasma- } \\
\text { zellen } \\
\text { teilweise arrangiert in den Kon- } \\
\text { turen von Lymphfollikeln } \\
\text { vermehrt Fibrozyten mit subtiler } \\
\text { Fibrose und Beimengung von } \\
\text { interstitiellen Makrophagen }\end{array}$ \\
\hline
\end{tabular}

Dermatitis. Auch in dem von uns vorgestellten Fall entsprach das histologische Bild diesen Beschreibungen. Tatsächlich konnte eine klinisch-histologische Analyse von 66 Fällen eines EAC zeigen, dass sich Fälle eines sogenannten EAC zumeist als id-Reaktion (spongiotische Dermatitis) infolge einer zu Grunde liegenden Dermatophytose entwickelten [9]. Im Gegensatz dazu sei der tiefe Typ mit seiner manschettenartigen Anordnung oberflächlicher und tiefer lymphozytärer Infiltrate, dem Vorhandensein von Melanophagen, der subtilen vakuolären Degeneration entlang der dermo-epidermalen Junktionszone und den individuellen nekrotischen Keratozyten charakteristisch für einen LE, insbesondere für einen Lupus tumidus [2].

Eine kürzliche Reevaluation von 90 klinisch und/oder histologisch diagnostizierten EAC-Fällen zeigte, dass es sich beim EAC nicht um eine eigenständige Entität, sondern um ein Reaktionsmuster handelt [5]. Im Ergebnis der klinisch-histologischen Reevaluation fanden sich entsprechend der klinischen Besonderheiten und des Musters und der Zusammensetzung des entzündlichen Infiltrats, abgesehen von einer Gruppe „Sonstiger Dermatosen“, die mit einem EAC im Einzellfall verwechselt werden können (Urtikaria, leukozytoklastische Vaskulitis, Arzneimittelexanthem, Psoriasis, Lymphom, Dermatomykose, Granuloma annulare sowie Insektenstichreaktion), drei Hauptgruppen von Erkrankungsbildern.

Die erste Gruppe umfasst Fälle eines tumiden LE und weiterer LEFormen. In diesen Fällen zeigt sich zumeist ein oberflächliches und tiefes, teilweise auch nur oberflächliches, betont ektatisch erweiterte Gefäße ummantelndes, lymphozytäres Infiltrat. Interface-Veränderungen fehlen oder sind nur minimal vorhanden. Klinisch finden sich einzelne bis wenige Läsionen zumeist am Rumpf, im Gesicht und an den Oberarmen jüngerer Frauen. Die sich peripher ausbreitenden, nicht-schuppenden Herde zeigen eine zentrale bräunliche bis gräulich-livide Verfärbung und leichte Atrophie. Die zweite Gruppe entspricht Fällen einer spongiotischen Dermatitis einschließlich einer irritierten oder exzessiven Pityriasis rosea. Neben Varianten einer PR finden sich in der Gruppe Fälle einer subakuten und chronischen Dermatitis im
Sinne eines „Ekzems“ wie zum Beispiel Stasis Dermatitis. Die Läsionen sind schuppig, oft multipel und häufiger an den unteren Extremitäten und dem angrenzenden Rumpf lokalisiert. Histologisch zeigen sich ein eher spärliches bis moderates superfizielles lymphozytäres Infiltrat mit gelegentlich einigen eosinophilen Granulozyten und Erythrozytenextravasaten sowie weiterhin Spongiose und Parakeratose. Die dritte Gruppe repräsentiert Fälle von Pseudolymphomen. Weder klinisch noch histologisch zeigen diese Fälle eine epidermale Beteiligung. Die Differenzialdiagnose zum tumiden LE ist schwierig. Auch Fälle einer „lymphocytic infiltration of the skin Jessner-Kanof" - selbst ein Sammelbegriff, hinter welchem sich neben Fällen eines tumiden LE und einer polymorphen Lichtdermatose auch Pseudolymphome finden [10-12] - zeigen ähnliche Veränderungen und bedingen Abgrenzungsschwierigkeiten. Histologisch präsentieren sich diese Fälle mit einem oberflächlichen und tiefen lymphozytären Infiltrat. In unterschiedlicher Anzahl sind Plasmazellen beigemischt. Interstitiell finden sich vermehrt Fibroblasten und einige Makrophagen. Klinisch weisen diese zur Migration tendierende, insgesamt etwas livid tingierte und an eine Sarkoidose erinnernde Herde mit bevorzugter Verteilung am Rumpf und den unteren Extremitäten keine zentrale Verfärbung auf. Das Ergebnis einer zusätzlichen immunhistochemischen Untersuchung mittels focus-floating microscopy (FFM) zeigte, dass sich in ca. $80 \%$ der als Pseudolymphom/vereinbar mit kutaner Lyme-Erkrankung interpretierten Fälle Spirochäten positiv färbten. Die Technik der FFM ist in anderen Arbeiten ausführlich beschrieben [13]. Im Unterschied zum einfachen Nachweis von Spirochäten der Gattung Treponema pallidum werden Borrelien im Gewebe gewöhnlich deutlich spärlicher angetroffen, zumeist verborgen im Netzwerk der Kollagenbündel. Das breite Spektrum an Hautveränderungen im Rahmen einer Borreliose ist nicht verwunderlich. Ähnlich der Multimorphologie der Syphilis zeigt auch die Lyme-Erkrankung vielzählige klinische Manifestationen [14]. Neben den klassischen klinischen Präsentationen können ungewöhnliche klinische Erscheinungen auftreten [15]. Auch einige bislang als unabhängig betrachtete klinische Krankheitsbilder werden heute mit 
einer Borrelien-Infektion assoziiert, wie die Morphea [16,17], der Lichen sclerosus [17], die Necrobiosis lipoidica [18], das nekrobiotische Xanthogranulom [19] und Fälle von Lymphocytic infiltration Jessner-Kanof $[10,12,20]$.

Zusammenfassend ist festzustellen, dass das EAC ein klinisches Reaktionsmuster und keine spezifische klinisch-histologische Entität ist. Hinter dem Begriff EAC verbergen sich verschiedene spezifische Dermatosen, vor allem Fälle eines LE und spongiotischer Dermatitiden vom Ekzemtyp und der PR, aber auch Pseudolymphome, darunter Borrelien-assoziierte. Die klinischen und histologischen Unterscheidungsmerkmale sind in $\bullet$ Tab. 1 zusammengefasst [5].

\section{Abkürzungen \\ $\nabla$ \\ B. burgdorferi - Borrelia burgdorferi \\ EAC - Erythema annulare centrifugum \\ FFM - focus-floating microscopy \\ LE - Lupus erythematosus \\ PR - Pityriasis rosea}

Interessenkonflikt: keiner vorhanden.

\section{Abstract}

\section{Erythema annulare centrifugum?}

Erythema annulare centrifugum (EAC) is a clinical reaction pattern and does not represent a specific clinicopathological entity. As the presented case of an EAC-like allergic contact dermatitis shows, behind the term EAC could be hidden various distinct diagnoses. In the meantime, this observation has been confirmed by an extensive retrospective analysis of so called EAC-cases. Thus, the clinical and histological differential diagnosis „EAC“ should lead the physician to consider three main groups of diseases which are often hidden behind. Besides (tumid) lupus erythematosus, this concerns spongiotic dermatitides either in terms of subacute and chronic dermatitis („eczema“), excessive variants of pityriasis rosea or stasis dermatitis. Moreover, less often, pseudolymphomas, in particular borrelia-associated pseudolymphomas are seen.

\section{Literatur}

1 Ackerman $A B$. Diagnosis of inflammatory skin diseases. Baltimore: Williams \& Wilkins, 1995

2 Weyers W, Diaz-Cascajo C, Weyers I. Erythema annulare centrifugum: results of a clinicopathologic study of 73 patients. Am J Dermatopathol 2003; 25: 451

3 Fritsch P. Dermatologie, Venerologie. Heidelberg: Springer, 2004

4 Freedberg IM, Eisen AZ, Wolff K, Austen KF, Goldsmith LA, Katz SI. Fitzpatrick's Dermatology in General Medicine. 6th edition. New York: McGraw-Hill, 2003

5 Ziemer M, Eisendle K, Zelger B. New concepts on erythema annulare centrifugum: a clinical reaction pattern that does not represent a specific clinicopathological entity. Br J Dermatol 2009; 160: 119

6 Darier J. De l'érythème annulaire centrifuge (érythème papulo-circiné migrateur et chronique) et de quelques eruptions analogues. Ann Dermatol Syph 1916; 6: 57-76

$7 \mathrm{Kerl} \mathrm{H}$, Garbe C, Cerroni L. Histopathologie der Haut. Heidelberg: Springer, 2003

8 Ackerman $A B$. Histologic Diagnosis of Inflammatory Skin Diseases. Philadelphia: Lea \& Febiger, 1978: 231

9 Kim KJ, Chang SE, Choi JH, Sung KJ, Moon KC, Koh JK. Clinicopathologic analysis of 66 cases of erythema annulare centrifugum. J Dermatol 2002; 29: 61

10 Ziemer M, Eisendle K, Muller H, Zelger B. Lymphocytic infiltration of the skin (Jessner-Kanof) but not reticular erythematous mucinosis occasionally represents clinical manifestations of Borrelia-associated pseudolymphoma. Br J Dermatol 2009; 161: 583

11 Toonstra J, Wildschut A, Boer J et al. Jessner's lymphocytic infiltration of the skin. A clinical study of 100 patients. Arch Dermatol 1989; 125: 1525

12 Abele DC, Anders $K H$, Chandler FW. Benign lymphocytic infiltration (Jessner-Kanof): another manifestation of borreliosis? J Am Acad Dermatol 1989; 21 (4 Pt 1): 795

13 Eisendle K, Grabner T, Zelger B. Focus floating microscopy: ,gold standard" for cutaneous borreliosis? Am J Clin Pathol 2007; 127: 213

14 Stechenberg $B W$. Lyme disease: the latest great imitator. Pediatr Infect Dis J 1988; 7: 402

15 Asbrink E, Hovmark A. Cutaneous manifestations in Ixodes-borne Borrelia spirochetosis. Int J Dermatol 1987; 26: 215

16 Eisendle K, Grabner T, Zelger B. Morphoea: a manifestation of infection with Borrelia species? Br J Dermatol 2007; 157: 1189

17 Tuffanelli $D$. Do some patients with morphea and lichen sclerosis et atrophicans have a Borrelia infection? Am J Dermatopathol 1987; 9: 371

18 Eisendle K, Baltaci M, Kutzner H, Zelger B. Detection of spirochaetal microorganisms by focus floating microscopy in necrobiosis lipoidica in patients from central Europe. Histopathology 2008; 52: 877

19 Zelger B, Eisendle K, Mensing C. Detection of spirochetal micro-organisms by focus-floating microscopy in necrobiotic xanthogranuloma. J Am Acad Dermatol 2007; 57: 1026

20 Kaatz M, Zelger B, Norgauer J, Ziemer M. Lymphocytic infiltration (Jessner-Kanof): lupus erythematosus tumidus or a manifestation of borreliosis? Br J Dermatol 2007; 157: 403 